\title{
A Conversation with Martin Hetzer
}

\author{
INTERVIEWER: Kim BAUMANN \\ Chief Editor, Nature Reviews Molecular Cell Biology
}

\begin{abstract}
Martin Hetzer is the Jesse and Caryl Philips Foundation Chair, a Professor in the Molecular and Cell Biology Laboratory, and Vice President and Chief Science Officer of the Salk Institute for Biological Studies in La Jolla, California.
\end{abstract}

Kim Baumann: One focus of your lab is long-lived proteins in the nucleus. Could you tell us how you discovered them and also what interests you in terms of the biology of these proteins?

Dr. Hetzer: It dates back to a study a postdoc performed in the lab in 2009 in nematodes-C. elegans - and he had a very basic question related to a subset of nuclear proteins called nucleoporins. They form the only gateway/ transport channel that mediates all molecular communication between the nucleus and the cytoplasm. He was interested in the question, "What happens to the expression of those genes that encode for proteins for the nuclear pore when cells leave the cell cycle and when they finally differentiate?"

Since the adult worm is entirely made of postmitotic or nondividing cells, he thought it was a great model system and he made a very surprising discovery. What he found is that a subset of nuclear pore proteins are present in the adult animal in every cell, very highly abundant, but the mRNA that encodes for those proteins is no longer detectable. He was very intrigued by that, because typically if a protein is present in a cell, then the mRNA is also present. Through further studies, he could show that all these nuclear pore complex proteins, which are large protein assemblies, are actually built during embryogenesis only in dividing cells in the embryo but no longer in the adult animals. The consequence of that was that those proteins have to last for the entire life span of the worm.

Kim Baumann: The nuclear pore is embedded in the nuclear envelope: that's the core of the pore, and then there are more peripheral structures. Is it true for all of the proteins that make the nuclear pores, or only for a subset?

Dr. Hetzer: Only for a subset of them. Every nuclear pore is made up of thirty different polypeptides and its only about half of them that form the scaffold structure of the nuclear pore that are no longer made. The other ones are still produced, so that is another level of complexity. Some are apparently very long-lived, while others are replaced and turn over quite rapidly.

The striking finding was that we had what we thought was the first example of a large protein assembly that lasts through the entire life span of the animal. Then, we thought, maybe that's specific for $C$. elegans, because the life span of a nematode is about two to three weeks. We wondered if this could be true for an animal that has a much longer life span. Could this also be true for humans? It's an interesting question, because many nondividing cells in adult organisms don't turn over. For instance, our neurons in our cortex and cerebellum are as old as we are. Could it be possible that this protein complex could actually last for years?

Kim Baumann: Are there are other long-lived proteins in tissues that have very low turnover, or is it specific to the nuclear pore?

Dr. Hetzer: We wondered the same thing. There are only a handful of proteins known to be extremely long-lived: Collagen or elastin - those are extracellular proteinsand $\beta$-crystallin, which forms the lenses of our eyes. Those are either outside of cells, or in very specialized environments.

We decided to do a pulse-chase labeling experiment in rodents. We fed the animals with a nonradioactive isotope, ${ }^{15} \mathrm{~N}$, chased with ${ }^{14} \mathrm{~N}$ for 2 years, and then performed quantitative mass spectrometry on isolated organs. We compared liver and brain initially. We did find about 50 very long-lived proteins. We've found, gratifyingly, the nuclear pore proteins again, very similar to what we saw in C. elegans. But, we also found proteins that are involved in gene regulation, such as histones and very specific histone variants. The most surprising part is we even found enzymes: We found histone deacyltransferase to be long-lived.

Kim Baumann: I would think that these kinds of proteins would be structural proteins, rather than involved in any regulation or regulatory processes. Is there any advantage to having them? Are there any common features?

Dr. Hetzer: There are no common features. There are no specific sequences. They can all be degraded in dividing cells, for instance. They are not necessarily structural proteins. We even first thought- for the nuclear pore, which is sort of a scaffold structure- that this would be an im-

(C) 2017 Hetzer. This article is distributed under the terms of the Creative Commons Attribution-NonCommercial License, which permits reuse and redistribution, except for commercial purposes, provided that the original author and source are credited. 
portant structural feature. It turns out that nuclear pores can actually form without those very long-lived components, so there must be another reason.

The best idea we have so far is for long-lived histones. We developed a system where you can distinguish between young and old protein in a differentiated cell in vitro. This trick allowed us to perform chromatin immunoprecipitation and genome sequencing. We found that long-lived histones are associated with repressed genes. One hypothesis that we are currently pursuing is that maybe the reason why very long-lived cells use long-lived histones is to reduce transcriptional noise. Whenever a nucleosome assembles, the cell risks that transcription factors might bind to the site and trigger transcription. It is well described across many species that, during aging, transcription noise increases. But a neuron in our brain has to stay in the same state. It always wants to be a neuron and perform its genetic program over decades. Maybe those cells use very, very long-lived proteins to very robustly repress genes.

Kim Baumann: You're saying that it's a way of stabilizing gene expression or regulating cell identity so that there is more stability.

Dr. Hetzer: Of course, we have no tests for this. One way to test this would be to force the turnover of those proteins and then we would expect transcription noise to increase during aging. That's one hypothesis. The other hypothesis that we are pursuing is linked to a phenomenon that was actually developed by French entomologists. It's something that I only recently learned and it's a phenomenon called "stigmergy." Most people I talk to have never heard of it. I'd never heard of it.

The principle is very interesting. There was a French entomologist who studied self-assembly in nature. He studied ants and termites and realized that insects, despite the fact that they have no intelligence, can produce a highly organized assembly. Termites can build buildings, but they also can self-assemble around a food source and different paths. He found that ants leave a pheromone trail to a food source. The next ant comes and leaves a stronger trail. They use this "stigmatus." So it's a "stigmergy" for self-assembly. One hypothesis that we are pursing is maybe the longevity of these proteins is part of that stigma: a guiding principle for the large protein assemblies. Even within a tissue, it might provide a principle- "the Stigmergy Principle" —of tissue assembly. You might have a subset of very long-lived proteins, or subcellular structures, or cells, to organize complex biological systems.

\section{Kim Baumann: Is this a way of stabilizing structures?}

Dr. Hetzer: It's more than stabilizing. It's really organizing. One outcome of our studies is that vasculature - capillary endothelial cells - are very, very old, even in organs such as the liver where hepatocytes turn over. So, maybe these long-lived cells are used to assemble and coordinate tissue separation within a tissue at the molecular level. Maybe these very stable nuclear pore proteins in the center are actually the organizing principle for the other compo- nents. That's possible, because the peripheral nuclear pore proteins actually do turn over and their residence time at the nuclear pore can be measured in seconds or minutes. You have six orders of magnitude difference in the time for which components are present in the same complex. That's unheard of. So, maybe you need that as sort of an organizing principle.

We have other ideas that are very speculative. For instance, in collagen and crystallin, people have identified chemical processes such as deamidation. Those are chemical reactions that can occur without any external factors and they cause changes in the amino acids such that even protein structure can change. Both occur naturally in longlived proteins. There are enzymes that can reverse that process, so perhaps there are processing places that allow proteins to change function over time. Perhaps even protein repair mechanisms are in place to allow those proteins to function over long periods of time. We're working on this, but we don't have a definitive answer.

Kim Baumann: Are these proteins subject to less damage so that they can be kept in a functional state longer? Is there any correlation between how much they could be exposed to damage and therefore they would be longlived?

Dr. Hetzer: That's a very good question. The extracellular proteins and the $\beta$-crystallin are really protected from cellular components, but these other long-lived proteins are fully exposed to potentially harmful metabolites. We do see signs of oxidative damage of those proteins. The problem is that we see the same level of damage in young animals and old animals, so we don't think that the functional decline is associated with damage. A still unexplained phenomenon is that we do see the specific loss of some of those long-lived proteins during aging and we don't know why.

Kim Baumann: Loss of the whole protein?

Dr. Hetzer: In the nuclear pore, for instance, it's specifically these very long-lived subcomplexes that are lost both in old neurons, and also in old long-lived pancreas cells. Maybe they're damaged and thus they are lost, or there is an age-dependent decline and a very, very slow replacement process. That's because they are maintained in a way that each individual component is slowly removed. We're talking about replacement rates for some of those, like, once in a lifetime for a mouse: very, very slow replacement. Since we know that protein homeostasis generally declines during aging, perhaps that's a failure to replace those components, but the jury's still out.

Kim Baumann: During aging, the machinery that ensures that there is a healthy protein declines. There is misfolding, or there is accumulation, or aggregation of proteins. Are any of these proteins involved in anything like that, given that they are possibly accumulating damage over time? Do they aggregate? Are they removed in the same ways that other proteins are removed?

Dr. Hetzer: We don't know the fate of those. We know that they disappear. Whether they aggregate some- 
where in a different cellular location, that's possible. We do see, as have many other people before us, an increase in aggregate formation in old cells, but whether they are accumulating in various aggregates or they are degraded once they are removed from the complex is an open question.

There is one link that is very interesting. One of the main functions of the nuclear pores is to form a barrier between the nucleoplasm and the cytoplasm, to only allow proteins and other molecules to pass that have a specific signal. In old cells, we found that other components can actually leak into the nucleus in an uncontrolled manner. One of those proteins is tubulin B3, a cytoplasmic protein; it should only be in the cytoplasm. We do see it inside the nuclei in old cells. In many cases, it forms very large aggregates, so they're not microtubules. They are large paracrystalline structures. We're currently analyzing the composition of those. Whether some of those long-lived proteins might actually be participating or enabling some of those aggregates is an open question.

Kim Baumann: What do you think is the next big question regarding these proteins? Are they still being identi- fied? Do we know how many of them there are, and in which places?

Dr. Hetzer: We are looking in different organs. We have comprehensively only analyzed the liver and the brain, but we are now analyzing the heart and we have identified a few. The really big question is: Are they potentially driving the aging process? Are they really important, or are they just like many other things in the cell that also start to fail. To test that, we face a very difficult problem. You can't use RNAi or any other method to deplete those proteins because, obviously, they are long-lived. We have to develop - which we are doing now - methods to extract those proteins from the complexes and trigger their turnover. When we can do this, we can go back into an animal and force the turnover and then ask if there is any consequence, either on longevity or survival of neurons. That is the biggest challenge. We're really tied into a causation potential between this longevity and age-dependent decline. That's the next big work. Another area that might be tied into this is protein repair mechanisms. These are very poorly understood. There might even be new biology involving proteins that might repair those long-lived proteins. 


\section{$\$_{\text {CSH\& }}^{\infty}$ Cold Spring Harbor Symposia SYMPOSIA}

\section{A Conversation with Martin Hetzer}

Cold Spring Harb Symp Quant Biol 2017 82: $394-396$ originally published online May 9, 2018 Access the most recent version at doi:10.1101/sqb.2017.82.034793

Creative This article is distributed under the terms of the

Commons http://creativecommons.org/licenses/by-nc/4.0/, which permits reuse and

License redistribution, except for commercial purposes, provided that the original author and source are credited.

Email Alerting Receive free email alerts when new articles cite this article - sign up in Service the box at the top right corner of the article or click here. 